\title{
Analysis of transient response of saturated porous elastic soil under cyclic loading using element-free Galerkin method
}

\author{
M.R. Karim ${ }^{\text {a }}$, T. Nogami ${ }^{\text {b }}$, J.G. Wang ${ }^{\text {a,* }}$ \\ a Tropical Marine Science Institute, Department of Civil Engineering, National University of Singapore, \\ 10 Kent Ridge Crescent, Singapore 119260, Singapore \\ ${ }^{\mathrm{b}}$ Department of Civil Engineering, National University of Singapore, Singapore 119260, Singapore
}

Received 31 October 2001; received in revised form 16 August 2002

\begin{abstract}
A two-dimensional numerical procedure is presented to analyse the transient response of saturated porous elastic soil layer under cyclic loading. The procedure is based on the element-free Galerkin method and incorporated into the periodic conditions (temporal and spatial periodicity). Its shape function is constructed by moving least-square approximants, essential boundary conditions are implemented through Lagrange multipliers and the periodic conditions are implemented through a revised variational formulation. Time domain is discretized through the Crank-Nicolson scheme. Analytical solutions are developed to assess the effectiveness and accuracy of the current procedure in one and two dimensions. For only temporal periodic problems, a one-dimensional transient problem of finite thickness soil layer is analysed for sinusoidal surface loading. For both temporal and spatial periodic problems, a typical two-dimensional wave-induced transient problem with the seabed of finite thickness is analysed. Finally, a moving boundary problem is analysed. It is found that the current procedure is simple, efficient and accurate in predicting the response of soil layer under cyclic loading.
\end{abstract}

(c) 2002 Elsevier Science Ltd. All rights reserved.

Keywords: Pore water pressure; Element-free Galerkin method; Moving least-square approximants; Lagrange multiplier; Cyclic loading; Periodic boundary

\section{Introduction}

Saturated soil can be idealised as two-phase media comprising deformable soil skeleton and pore fluid. The transient response of the saturated soil is especially important to understand the deformations and the pore water pressures generated by ground motion. This response is a key factor to the analyses of buildings, machine foundation, offshore structures, wave propagation in geological medium due to blast or earthquake, and pile driving. A fluid-filled porous medium theory was proposed by Biot (1941) to analyze this transient response. Most of the transient response problems are solved by numerical methods such as finite

\footnotetext{
${ }^{*}$ Corresponding author. Tel.: +65-6874-6591; fax: +65-6779-1635.

E-mail address: tmswjg@nus.edu.sg (J.G. Wang).
} 
element method (FEM) and finite difference method (FDM) in conjunction with appropriate time integration schemes. Typical FEM methods were proposed by Sandhu and Wilson (1969), Ghaboussi and Wilson (1972, 1973), Ghaboussi and Dikman (1978), Prevost (1982), Zeinkiewicz et al. (1977), Zeinkiewicz (1980) and Zeinkiewicz and Shiomi (1984). However, it is difficult for FEM to analyze the problems associated with moving boundary. A meshless method is an effective alternative.

This paper will study the transient response of seabed under wave loading using meshless element-free Galerkin method (EFGM). The wave-induced transient response has its own characteristics: first, wave loading is applied on the surface of seabed through water pressure. If shallow water is concerned, this wave loading still has energy to produce impact on the seabed. When water wave propagates over seabed, a cyclic pressure will exert on the surface of seabed, thus causing pore fluid in the seabed to flow and seabed to deform. Transient fluctuation of pore water pressure generates the transient reduction of effective stress, and thus seabed may lose its strength momentarily during a cyclic wave. Second, water over the seabed and pore water in seabed interact each other. The soil of the seabed has an effect on the wave train. This interaction also makes the water-seabed interface complicated. Furthermore, an object placed on the seabed may move along the seabed surface. This is a moving boundary problem.

Several publications are available for the wave-induced pore pressures and effective stresses analysis. Most of these are based on Biot's consolidation theory (1941). The approaches can be roughly classified into two categories: analytical approaches and numerical methods. Among analytical approaches, Yamamoto et al. (1978) and Madsen (1978) considered compressible pore fluid in a porous seabed of infinite thickness subjected to a two-dimensional wave. Their final governing equation, for saturated isotropic soils, was a sixth-order linear differential equation. Later Okusa (1985) reduced the order of the governing equation to fourth order. However, they assumed periodic variables in time domain and in space, and thus the solution is not general. As the governing equations are generally difficult to solve analytically for finite soil thickness, Thomas (1988) developed a semi-analytical one-dimensional finite element procedure to simulate the waveinduced stresses and pore water pressures. He also assumed that all variables are harmonic. His formulation was tremendously complicated. Among numerical algorithms, FEM and FDM were main tools. Although they are successful in many problems, some difficulties remain in the treatment of mesh distortion associated with moving boundary conditions. Recently developed meshless methods could overcome these disadvantages because meshless methods do not use any element. On this meaning, meshless methods are attractive for the transient analysis of wave-induced seabed responses.

A two-dimensional transient problem under wave-induced load has essential and natural boundaries that are periodic in time and in space. The periodic temporal boundary conditions are easily implemented in numerical procedures. However, a special procedure is required for the implementation of boundary conditions with periodicity in space. This paper will develop a variational approach to treat periodic temporal and spatial boundary conditions, which are common boundaries in those transient problems under cyclic loading.

Meshless methods are recently developed numerical techniques. EFGM proposed by Belytschko et al. (1994) is a successful meshless technique that requires only nodes to discretize a problem domain. Its shape functions are constructed by moving least-square (MLS) approximants (Lancaster and Salkauskas, 1981). Other forms of meshless methods include reproducing kernel particle method (Liu et al., 1995), $h-p$ clouds (Duarte and Oden, 1996), the partition of unity (Babuska and Melenk, 1997), smooth particle hydrodynamics (Monaghan, 1988), and radial point interpolation method (Wang and Liu, 2002).

This paper presents a meshless element-free Galerkin procedure to solve the transient response of fluidsaturated porous elastic soil under cyclic loading. The EFGM is firstly applied to a one-dimensional problem. This is a typical temporally periodic problem to investigate the transient response of a fully saturated, elastic and isotropic porous soil layer subjected to sinusoidal surface loading. A two-dimensional wave-induced transient problem for a seabed with finite thickness is studied when subjected to a progressive wave. This problem includes both temporal and spatial periodic boundary conditions. A moving boundary 
problem is also designed to check the capability of the current procedure. This paper is organised as follows. First, brief descriptions of MLS approximants are presented. Then, governing equations for fluid-saturated elastic-porous medium are described. Periodic temporal and spatial boundary conditions are incorporated into the variational formulations. The numerical implementation for above variational formulations is presented. Finally, examples are used to assess the performance of the current procedure.

\section{Moving least-square approximation}

MLS method is employed in the EFGM to approximate a function $u(\bar{x})$ with $u^{h}(\bar{x})$, where $u(\bar{x})$ is the actual function and $u^{h}(\bar{x})$ is its approximation. The approximation consists of three components: a basis, usually a polynomial; a weight function associated with each node; and a set of coefficients that depend on node position. The weight function is non-zero only over a small sub-domain around the node. This nonzero domain is called compact support or domain of influence. The overlap of the nodal domains of influence defines a nodal connectivity.

\subsection{Moving least-square approximants}

The MLS approximant (Lancaster and Salkauskas, 1981) $u^{h}(\bar{x})$ is posed as follows:

$$
u^{h}(\bar{x})=\sum_{j=0}^{m} p_{j}(\bar{x}) a_{j}(\bar{x}) \equiv p^{\mathrm{T}}(\bar{x}) a(\bar{x})
$$

where $p_{j}(\bar{x})$ is monomial in the space co-ordinates, $\bar{x}^{\mathrm{T}}=[x, z]$, and $a_{j}(\bar{x})$ its coefficient. In a complete polynomial of order $k$ (including $m$ terms), $p_{j}(\bar{x})$ and $a_{j}(\bar{x})$ are given by

$$
\begin{aligned}
& p^{\mathrm{T}}(\bar{x})=\left[\begin{array}{llllllll}
1 & x & z & x^{2} & x z & z^{2} & \ldots & z^{k}
\end{array}\right] \\
& a^{\mathrm{T}}(\bar{x})=\left[\begin{array}{lllll}
a_{0}(\bar{x}) & a_{1}(\bar{x}) & \ldots & a_{m}(\bar{x})
\end{array}\right]
\end{aligned}
$$

At each point $\bar{x}, a_{j}(\bar{x})$ is so chosen as to minimize the weighted residual $L_{2}$-norm:

$$
J=\sum_{I=1}^{n} w\left(\bar{x}-\bar{x}_{I}\right)\left[p^{\mathrm{T}}(\bar{x}) a(\bar{x})-u_{I}\right]^{2}
$$

where $n$ is the number of nodes $I$ in the neighbourhood of $\bar{x}$ for which the weight function $w\left(\bar{x}-\bar{x}_{I}\right) \neq 0$, and $u_{I}$ refers to the nodal index of $u$ at $\bar{x}=\bar{x}_{I}$. The minimum of $J$ with respect to $a(\bar{x})$ gives

$$
a(\bar{x})=A^{-1}(\bar{x}) B(\bar{x}) u
$$

where $A=p^{\mathrm{T}} w(\bar{x}) p$ and $B=p^{\mathrm{T}} w(\bar{x})$.

Therefore, the approximation is obtained as

$$
u^{h}(\bar{x})=\sum_{I=1}^{n} \sum_{j=0}^{m} p_{j}(\bar{x})\left(A^{-1}(\bar{x}) B(\bar{x})\right)_{j I} u_{I}=\sum_{I=1}^{n} \phi_{I}(\bar{x}) u_{I}
$$

where the shape function $\phi_{I}(\bar{x})$ is defined by

$$
\phi_{I}(\bar{x})=\sum_{j=0}^{m} p_{j}(\bar{x})\left(A^{-1}(\bar{x}) B(\bar{x})\right)_{j I}=p^{\mathrm{T}} A^{-1} B_{I}
$$


Their spatial derivatives are obtained as

$$
\phi_{I, \bar{x}}=\left(p^{\mathrm{T}} A^{-1} B_{I}\right)_{, \bar{x}}
$$

or

$$
\phi_{I, \bar{x}}=p_{, \bar{x}}^{\mathrm{T}} A^{-1} B_{I}+p^{\mathrm{T}}\left(A^{-1}\right)_{, \bar{x}} B_{I}+p^{\mathrm{T}} A^{-1} B_{I, \bar{x}}
$$

where

$$
B_{I, \bar{x}}(\bar{x})=\frac{\mathrm{d} w\left(\bar{x}-\bar{x}_{I}\right)}{\mathrm{d} \bar{x}} p\left(\bar{x}_{I}\right), \quad A_{\bar{x}}=\sum_{I=1}^{n} \frac{\mathrm{d} w\left(\bar{x}-\bar{x}_{I}\right)}{\mathrm{d} \bar{x}} p\left(\bar{x}_{I}\right) p^{\mathrm{T}}\left(\bar{x}_{I}\right), \quad\left(A^{-1}\right)_{\bar{x}}=-A^{-1} A_{\overline{,}} A^{-1}
$$

\subsection{Weight functions}

Weight function $w_{I}(\bar{x}) \equiv w\left(\bar{x}-\bar{x}_{I}\right)$ plays an important role in the EFGM. The weight function should be non-zero only over a small neighbourhood of $\bar{x}_{I}$. Usually, $w_{I}(\bar{x}) \equiv w\left(\bar{x}-\bar{x}_{I}\right)=w_{I}\left(d_{I}\right)$, where $d_{I}=\left\|\bar{x}-\bar{x}_{I}\right\|$ is the distance between the two points $\bar{x}_{I}$ and $\bar{x}$. The $w\left(\bar{x}-\bar{x}_{I}\right)$ should be smooth enough. If $w\left(\bar{x}-\bar{x}_{I}\right)$ is $C^{1}$ continuous, and a linear polynomial basis is used, the shape function $\phi_{I}(\bar{x})$ would be $C^{1}$ continuous (Lancaster and Salkauskas, 1981). Cubic spline weight function is considered in the present formulation:

$$
w\left(\bar{x}-\bar{x}_{I}\right)= \begin{cases}\frac{2}{3}-4\left(\frac{d_{I}}{d_{m I}}\right)^{2}+4\left(\frac{d_{I}}{d_{m I}}\right)^{3}, & \text { for }\left(\frac{d_{I}}{d_{m I}}\right) \leqslant \frac{1}{2} \\ \frac{4}{3}-4\left(\frac{d_{I}}{d_{m I}}\right)+4\left(\frac{d_{I}}{d_{m I}}\right)^{2}-\frac{4}{3}\left(\frac{d_{I}}{d_{m I}}\right)^{3}, & \text { for } \frac{1}{2}<\left(\frac{d_{I}}{d_{m I}}\right) \leqslant 1 \\ 0, & \text { for }\left(\frac{d_{I}}{d_{m I}}\right)>1\end{cases}
$$

where $d_{m I}$ is the size of the compact support of $\bar{x}_{I}$. Generally $d_{m I}=d_{\max } c_{I}$. The $d_{\max }$ is a scaling parameter known as support size factor. The $c_{I}$ is the maximum distance between two neighbouring nodes in each direction (Dolbow and Belytschko, 1998). Circular or square domains of influence are usually used in practice.

\subsection{Enforcement of essential boundary conditions}

MLS shape functions do not satisfy the Kronecker delta criterion: $\phi_{I}\left(\bar{x}_{j}\right) \neq \delta_{i j}$. That means the MLS approximants are not equal to the true function at nodes unless the weight function is singular, i.e., $u^{h}(\bar{x}) \neq u_{I}$. In other words, the approximation at the $I$ th node depends on the nodal index $u_{I}$ as well as other nodal indices within the domain of influence of node $I$. Although several techniques have been developed to enforce essential boundary conditions, this paper will use Lagrange multiplier method as an easy and direct method to impose essential boundary conditions (Belytschko et al., 1996).

\section{Weak form and numerical implementation}

\subsection{Governing equations}

Soil skeletons are assumed to be elastic, isotropic and homogeneous. Pore water flows follow Darcy's law. Body forces as well as compressibility of the pore water are also taken into consideration. The transient 
response of porous medium under wave-induced loading is generally described by Biot's consolidation theory (1941) and Verruijt's storage equation (1969), which are expressed respectively as follows:

$$
\begin{aligned}
& D \partial^{2} u+\partial_{\mathrm{s}} p+b=0 \\
& \partial_{\mathrm{s}}\left(\frac{\partial u}{\partial t}\right)-n \beta \frac{\partial p}{\partial t}-\frac{k}{\gamma_{\mathrm{w}}} \partial_{\mathrm{s}}^{2} p=0
\end{aligned}
$$

where $u$ is the displacement; $p$, the pore water pressure; $b$, the body force vector; $n$, the porosity of soil skeleton; $k$, the soil permeability; $\beta$, the compressibility of pore fluid; and $\gamma_{\mathrm{w}}$, the unit weight of pore fluid; $t$, the real time. For a plane strain problem,

$$
D=\frac{E}{(1+v)(1-2 v)}\left[\begin{array}{ccc}
1-v & v & 0 \\
v & 1-v & 0 \\
0 & 0 & \frac{1-2 v}{2}
\end{array}\right], \quad \partial=\left[\begin{array}{cc}
\frac{\partial}{\partial x} & 0 \\
0 & \frac{\partial}{\partial z} \\
\frac{\partial}{\partial z} & \frac{\partial}{\partial x}
\end{array}\right], \quad \partial_{\mathrm{s}}=\left[\begin{array}{c}
\frac{\partial}{\partial x} \\
\frac{\partial}{\partial z}
\end{array}\right]
$$

The effective stress is expressed in terms of displacement as

$$
\sigma^{\prime}=D \partial u
$$

\subsection{Boundary conditions}

Boundary conditions include soil skeleton and fluid boundaries as

$$
\begin{aligned}
& u(x, t)=\bar{u}(x, t) \quad \text { on } \Gamma_{u} \\
& p(x, t)=\bar{p}(x, t) \quad \text { on } \Gamma_{\mathrm{p}} \\
& \sigma \cdot \hat{n}(x, t)=\bar{t}(x, t) \quad \text { on } \Gamma_{\sigma} \\
& \frac{k}{\gamma_{\mathrm{w}}} \frac{\partial p}{\partial \hat{n}}(x, t)=\bar{\varphi}(x, t) \quad \text { on } \Gamma_{\varphi}
\end{aligned}
$$

$\bar{\varphi}$ and $\bar{t}$ indicate pore water flux and traction, respectively. $\hat{n}$ is the unit normal to boundary $\Gamma_{\sigma} . \Gamma_{u}, \Gamma_{\mathrm{p}}, \Gamma_{\sigma}$ and $\Gamma_{\varphi}$ are the boundaries where displacement, pore water pressure, total stress and flux of pore water are prescribed. Obviously, they satisfy following relations:

$$
\begin{aligned}
& \Gamma_{u} \cup \Gamma_{\sigma}=\Gamma \quad \text { and } \quad \Gamma_{u} \cap \Gamma_{\sigma}=\emptyset \\
& \Gamma_{\mathrm{p}} \cup \Gamma_{\varphi}=\Gamma \quad \text { and } \quad \Gamma_{\mathrm{p}} \cap \Gamma_{\varphi}=\emptyset
\end{aligned}
$$

where $\Gamma$ is the boundary of problem domain.

\subsection{Periodic boundary conditions}

Temporal periodic conditions are for one-dimensional analysis, while spatial and temporal periodic boundary conditions are for multi-dimensional analysis. For example, when a sinusoidal traction propagates over the seabed surface, the soil response is spatially periodic. For such a problem, a certain length of soil mass along the horizontal direction is analysed. This length is equal to at least one wavelength of cyclic 
loading. Such an analysis model creates two virtual boundaries at both ends, denoted as $\Gamma_{\mathrm{vl}}$ and $\Gamma_{\mathrm{vr}}$. Because soil responses are identical at these virtual boundaries, the spatial periodic conditions can be expressed as:

$$
\begin{aligned}
& u^{\mathrm{vl}}=u^{\mathrm{vr}} \\
& p^{\mathrm{vl}}=p^{\mathrm{vr}}
\end{aligned}
$$

where $u^{\mathrm{vl}}, u^{\mathrm{vr}}$ and $p^{\mathrm{vl}}, p^{\mathrm{vr}}$ are the displacements and pore water pressures on the left and right side virtual boundaries, respectively. Eqs. (14a) and (14b) are new boundary conditions and will be incorporated into the variational formulations.

\subsection{Variational formulations}

The variational (or weak) forms for Eqs. (10) and (11) and (13a)-(13d), (14a), (14b) are formulated with trial functions of $u(\bar{x}, t)$ and $p(\bar{x}, t)$, Lagrange multipliers $\lambda_{1}(\bar{x}, t), \lambda_{2}(\bar{x}, t), \lambda_{3}(\bar{x}, t)$ and $\lambda_{4}(\bar{x}, t)$ and their corresponding test functions $\delta v(\bar{x}, t), \delta p(\bar{x}, t), \delta \lambda_{1}(\bar{x}, t), \delta \lambda_{2}(\bar{x}, t), \delta \lambda_{3}(\bar{x}, t)$ and $\delta \lambda_{4}(\bar{x}, t)$. Trial functions do not satisfy essential boundary conditions so that they are imposed with Lagrange multipliers. The subscripts ' 1 ' and ' 2 ' stand for the displacement and the pore water pressure on respective boundaries $\left(\Gamma_{u}\right.$ and $\left.\Gamma_{\mathrm{p}}\right)$. The subscripts ' 3 ' and ' 4 ' stand for the displacement and the pore water pressure on virtual boundaries $\Gamma_{\mathrm{v} u}$ and $\Gamma_{\mathrm{vp}}$. A weighted residual form for these equations is obtained as follows:

$$
\begin{aligned}
& \int_{\Omega} \delta v\left(D \partial^{2} u+\partial_{\mathrm{s}} p+b\right) \mathrm{d} \Omega+\int_{\Omega} \delta p\left\{\partial_{\mathrm{s}}\left(\frac{\partial u}{\partial t}\right)-n \beta \frac{\partial p}{\partial t}-\frac{k}{\gamma_{\mathrm{w}}} \partial_{\mathrm{s}}^{2} p\right\} \mathrm{d} \Omega+\int_{\Gamma_{u}} \delta v_{u}(u-\bar{u}) \mathrm{d} \Gamma \\
& +\int_{\Gamma_{\sigma}} \delta v_{\sigma}(\sigma \cdot \hat{n}-\bar{t}) \mathrm{d} \Gamma+\int_{\Gamma_{\mathrm{p}}} \delta p_{\mathrm{p}}(p-\bar{p}) \mathrm{d} \Gamma+\int_{\Gamma_{\varphi}} \delta p_{\varphi}\left(\frac{k}{\gamma_{\mathrm{w}}} \frac{\partial p}{\partial \hat{n}}-\bar{\varphi}\right) \mathrm{d} \Gamma \\
& \quad+\left(\int_{\Gamma_{\mathrm{vl}}} \delta v_{\mathrm{v} u} u \mathrm{~d} \Gamma-\int_{\Gamma_{\mathrm{vr}}} \delta v_{\mathrm{v} u} u \mathrm{~d} \Gamma\right)+\left(\int_{\Gamma_{\mathrm{vl}}} \delta p_{\mathrm{vp}} p \mathrm{~d} \Gamma-\int_{\Gamma_{\mathrm{vr}}} \delta p_{\mathrm{vp}} p \mathrm{~d} \Gamma\right)=0
\end{aligned}
$$

Integrating by parts the first two terms of first integral and third term of the second integral and choosing $\delta v_{\sigma}=-\delta v$ and $\delta p_{\phi}=-\delta p$, Eq. (15) is partitioned into six equations. Defining $\lambda_{1}=\sigma \cdot \hat{n}$ on $\Gamma_{\mathrm{u}}$, $\lambda_{2}=\left(k / \gamma_{\mathrm{w}}\right)(\partial p / \partial \hat{n})$ on $\Gamma_{\mathrm{p}}$ and the associated trial functions as $\delta \lambda_{1}=\delta v_{u}, \delta \lambda_{2}=\delta p_{\mathrm{p}}, \delta \lambda_{3}=\delta v_{\mathrm{v} u}$ and $\delta \lambda_{4}=\delta p_{\mathrm{vp}}$, Eq. (15) becomes (see Modaressi and Aubert, 1996 for further details):

$$
\begin{aligned}
\int_{\Omega} D \partial u \cdot \partial \delta v \mathrm{~d} \Omega+\int_{\Omega} p \cdot \partial_{\mathrm{s}} \delta v \mathrm{~d} \Omega= & \int_{\Gamma_{\sigma}} \bar{t} \delta v \mathrm{~d} \Gamma-\int_{\Gamma_{u}} \lambda_{1} \delta v \mathrm{~d} \Gamma+\int_{\Omega} b \cdot \delta v \mathrm{~d} \Omega \\
& -\left(\int_{\Gamma_{\mathrm{vl}}} \lambda_{3} \delta v \mathrm{~d} \Gamma-\int_{\Gamma_{\mathrm{vr}}} \lambda_{3} \delta v \mathrm{~d} \Gamma\right) \\
\int_{\Omega} \delta p \cdot \partial_{\mathrm{s}}\left(\frac{\partial u}{\partial t}\right) \mathrm{d} \Omega-\int_{\Omega} n \beta \delta p \cdot \frac{\partial p}{\partial t} \mathrm{~d} \Omega+\int_{\Omega} \frac{k}{\gamma_{\mathrm{w}}} \partial_{\mathrm{s}} p \cdot \partial_{\mathrm{s}} \delta p \mathrm{~d} \Omega=\int_{\Gamma_{\varphi}} \delta p \bar{\varphi} \mathrm{d} \Gamma-\int_{\Gamma_{\mathrm{p}}} \lambda_{2} \delta p \mathrm{~d} \Gamma & -\left(\int_{\Gamma_{\mathrm{vl}}} \lambda_{4} \delta p \mathrm{~d} \Gamma-\int_{\Gamma_{\mathrm{vr}}} \lambda_{4} \delta p \mathrm{~d} \Gamma\right) \\
\int_{\Gamma_{u}} \delta \lambda_{1} u \mathrm{~d} \Gamma=\int_{\Gamma_{u}} \delta \lambda_{1} \bar{u} \mathrm{~d} \Gamma &
\end{aligned}
$$




$$
\begin{aligned}
& \int_{\Gamma_{\mathrm{p}}} \delta \lambda_{2} p \mathrm{~d} \Gamma=\int_{\Gamma_{\mathrm{p}}} \delta \lambda_{2} \bar{p} \mathrm{~d} \Gamma \\
& \int_{\Gamma_{\mathrm{vl}}} \delta \lambda_{3} u \mathrm{~d} \Gamma=\int_{\Gamma_{\mathrm{vr}}} \delta \lambda_{3} u \mathrm{~d} \Gamma \\
& \int_{\Gamma_{\mathrm{vl}}} \delta \lambda_{4} p \mathrm{~d} \Gamma=\int_{\Gamma_{\mathrm{vr}}} \delta \lambda_{4} p \mathrm{~d} \Gamma
\end{aligned}
$$

\subsection{Discrete equations}

The MLS approximant in Eq. (6) is used to discretize the spatial variables in the weak form in Eqs. (16a)-(16f). The spatial approximants are as follows:

$$
\begin{aligned}
& u(\bar{x})=\sum_{I=1}^{n} \phi_{I} u_{I} \\
& p(\bar{x})=\sum_{I=1}^{n} \phi_{I} p_{I} \\
& \lambda_{1}(\bar{x})=\sum_{I=1}^{m} N_{I} \lambda_{1 I} \\
& \lambda_{2}(\bar{x})=\sum_{I=1}^{m} N_{I} \lambda_{2 I} \\
& \lambda_{3}(\bar{x})=\sum_{I=1}^{m} N_{I} \lambda_{3 I} \\
& \lambda_{4}(\bar{x})=\sum_{I=1}^{m} N_{I} \lambda_{4 I}
\end{aligned}
$$

In Eqs. (17a)-(17f) $N_{I}$ is a shape function of Lagrange interpolant and $m$ is the node number in influence domain. Time integration scheme is generally expressed for any function $f(t)$ as

$$
\int_{t}^{t+\Delta t} f(t) \mathrm{d} t=\Delta t[\theta f(t+\Delta t)+(1-\theta) f(t)]
$$

where $0 \leqslant \theta \leqslant 1$, and $t$ and $\Delta t$ denote the real time and its increment, respectively. Therefore, the final discrete equations are obtained as following matrix form:

$$
[R]\left[S^{t+1}\right]=[F]+[Q]\left[S^{t}\right]
$$

where $[R],\left[S^{t+1}\right],[F],[Q]$ and $\left[S^{t}\right]$ are given in Appendix A. It is noted that the $[R]$ and $[Q]$ are the function of parameter $\theta$. When $\theta=0.5$, the time integration is Crank-Nicolson scheme (Wang et al., 2002; Karim et al., 2001). 


\subsection{Numerical implementation}

The implementation procedure for above numerical model is as follows:

1. Define geometrical dimensions and material properties of the domain $\Omega$.

2. Choose basis and weight functions for MLS approximants.

3. Set up nodal coordinates and determine domain of influence for each node.

4. Define integration cells and determine Gauss points for each integration cell $\Omega_{i}$ and boundary cell $\Gamma_{u i}$, $\Gamma_{\mathrm{p} i}, \Gamma_{\mathrm{t} i}$ and $\Gamma_{\varphi i}$. Set up their weights and Jacobian.

5. Loop over Gauss points:

a. Determine neighbouring nodes of each Gauss point.

b. Compute shape function and derivatives at the Gauss points.

c. Assemble matrix $B_{I}, A_{J}, K_{I J}, L_{I J}, M_{I J}$ and $H_{I J}$ (Appendix A) to compute $R$ and $Q$.

d. End Gauss point loop.

6. Integrate along boundaries to get the $f_{u I}, f_{p I}, G_{I K}, G_{I K}^{\prime}, G_{I K}^{\mathrm{vl}}, G_{I K}^{\mathrm{vr}}, G_{I K}^{\mathrm{vl}}, G_{I K}^{\mathrm{vr}}, f_{\lambda_{1} I}$ and $f_{\lambda_{2} I}$ (Appendix A) to compute $F$ matrix.

7. Solve the system equation of Eq. (19) to get nodal indices of $u$ and $p$. Determine displacements and pore water pressures at each node using Eqs. (17a) and (17b).

8. Determine effective stress $\sigma^{\prime}$ at each Gauss point through Eq. (12).

\section{Assessment of the current numerical procedure}

Typical one- and two-dimensional problems are studied for the transient response of soil mass under cyclic loading. A two-dimensional computer code is made for a plane strain problem. Numerical model parameters are listed as follows: linear basis function $(m=3)$ in Eq. (2), cubic spline weight expressed in Eq. (9) with $d_{\max }=2.5$. The domain is discretized with regularly distributed nodes for approximation and regular background cells for integration. Gauss quadrature with $4 \times 4$ points is used for the integration of the Galerkin weak form. Linear Gauss quadrature with four points is used for the line integration of the $f_{\mathrm{p}}$, $f_{\lambda_{1}}, f_{\lambda_{2}}$ vectors and $G, G^{\prime}, G^{\mathrm{vl}}, G^{\mathrm{vr}}, G^{\prime \mathrm{vl}}, G^{\prime \mathrm{vr}}$ matrices along essential boundaries, as well as force $f_{u}$ vector along traction boundaries. The time integration parameter $\theta$ is taken as 0.5 .

\subsection{One-dimensional transient soil response under cyclic loading}

Fig. 1 is the problem where cyclic loading is $100 \sin (\varpi t) \mathrm{kN} / \mathrm{m}^{2}$ and the $\varpi$ is the angular frequency. If the load period is taken as $20 \mathrm{~s}$, the frequency $\varpi$ is $0.31416 \mathrm{rad} / \mathrm{s}$. The load on the soil surface is assumed to last for $240 \mathrm{~s}$ (12 cycles). Body force of the soil is not taken into consideration.

The soil thickness $(h)$ is assumed to be $20 \mathrm{~m}$. Soil is isotropic and elastic with Young's modulus, $E=2500 \mathrm{kN} / \mathrm{m}^{2}$; Poisson's ratio, $v=0.3$; porosity, $n=0.4$; isotropic permeability, $k=2.5 \times 10^{-2} \mathrm{~m} / \mathrm{s}$. Water parameters are: density, $\gamma_{\mathrm{w}}=10 \mathrm{kN} / \mathrm{m}^{3}$; compressibility, $\beta=4.1 \times 10^{-6} \mathrm{~m}^{2} / \mathrm{kN}$. The boundary conditions are as follows: topsoil surface is completely permeable $(p=0)$ and the bottom is rigid $\left(u_{z}=0\right)$ and impermeable $\left(\partial_{n} p=0\right)$. Only temporal periodic boundary conditions are needed for this problem. There are no virtual boundaries $\Gamma_{\mathrm{vl}}$ and $\Gamma_{\mathrm{vr}}$. This leads to the simplification of various elements of Eq. (19) as given in Appendix A.

Fig. 2 gives the physical model and its meshless model. The soil column is of unit width $(L=1 \mathrm{~m})$ and of the thickness of $h=20 \mathrm{~m}$. There are no flow and movement along the horizontal direction as shown in Fig. 2(a). Eighty-two (82) equally spaced nodes as shown in Fig. 2(b) are included in the meshless model, where background cells for integration are enclosed by dotted lines. In order to simulate periodic loading, time 


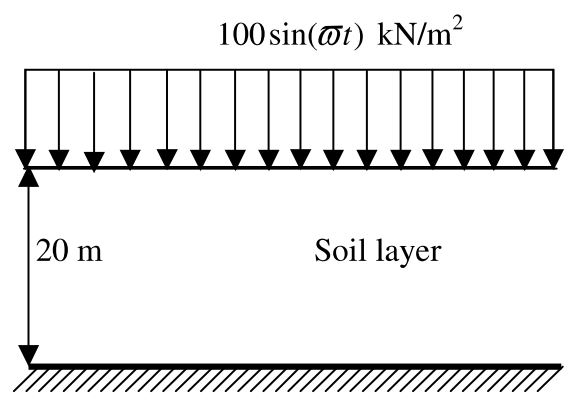

(a)

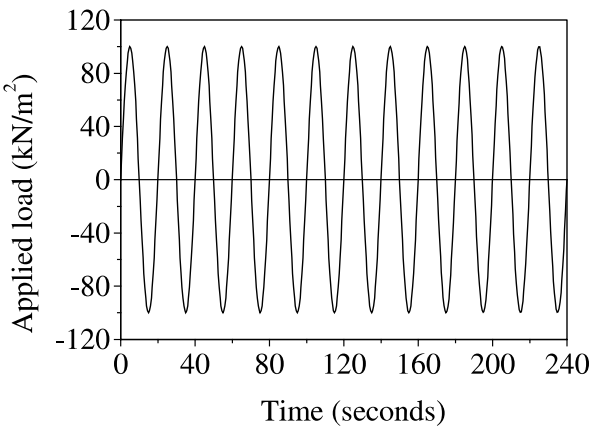

(b)

Fig. 1. Typical one-dimensional problem subjected to cyclic loading. (a) Problem description and (b) load history.
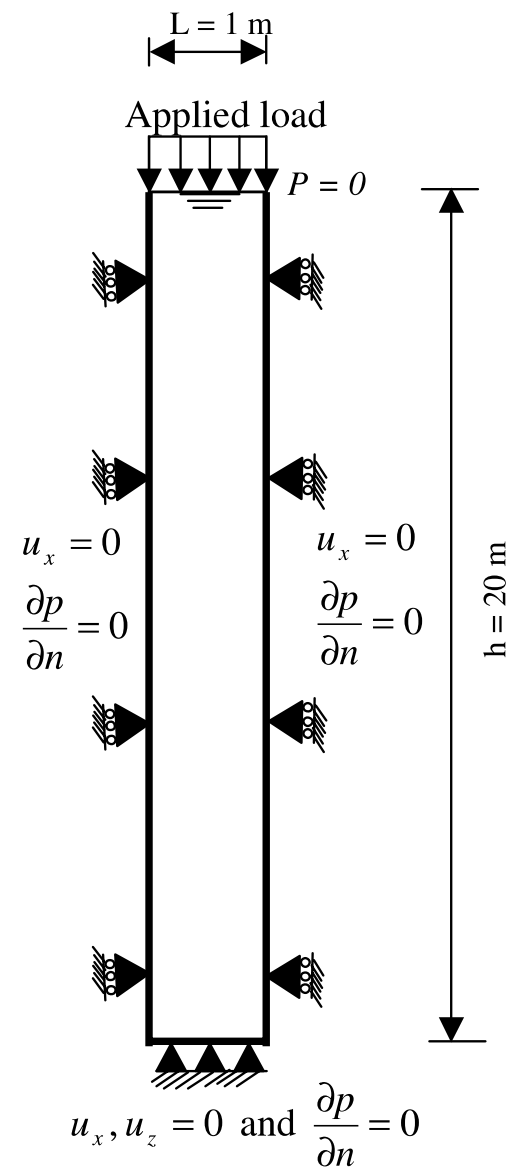

(a)

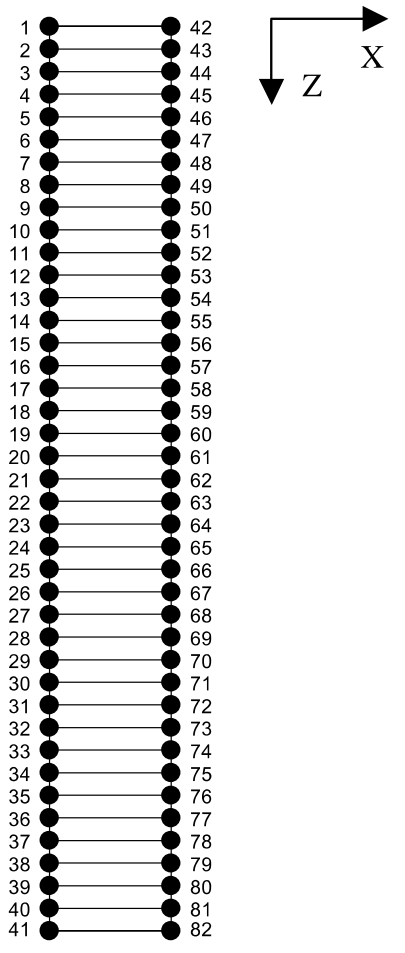

(b)

Fig. 2. 1-D model and its meshless scheme. (a) 1-D model and (b) node distribution. 
step size is so selected that it is a multiple of the load period. A time increment of $0.0125 \mathrm{~s}$ is used until 0.125 $\mathrm{s}$ and for the remaining time step size is chosen as $0.125 \mathrm{~s}$. Such a choice has a total of 2410 time steps within the $240 \mathrm{~s}$.

An analytical solution is obtained from the frequency domain analysis of the governing equations of Eqs. (10) and (11). The analytical solutions for displacement $(u)$, pore water pressure $(p)$ and effective stress $\left(\sigma^{\prime}\right)$ are obtained as

$$
\begin{aligned}
& p=-\operatorname{Im}\left\{\left(F+G \mathrm{e}^{-a z}+H \mathrm{e}^{a z}\right) \mathrm{e}^{-\mathrm{i} \varpi t}\right\} \\
& u=-\operatorname{Im}\left\{\left(-n \beta F z+\frac{R}{a} G \mathrm{e}^{-a z}-\frac{R}{a} H \mathrm{e}^{a z}-T\right) \mathrm{e}^{-\mathrm{i} \varpi t}\right\} \\
& \sigma^{\prime}=-\operatorname{Im}\left\{\left(\frac{n \beta}{m_{\mathrm{v}}} F+\frac{R}{m_{\mathrm{v}}} G \mathrm{e}^{-a z}+\frac{R}{m_{\mathrm{v}}} H \mathrm{e}^{a z}\right) \mathrm{e}^{-\mathrm{i} \varpi t}\right\}
\end{aligned}
$$

where $z$ is measured from seabed surface. Other constants in Eqs. (20a)-(20c) are given by

$$
\begin{aligned}
a & =a_{1}-\mathrm{i} a_{2} \quad \text { and } a_{1}=a_{2}=\sqrt{\frac{\varpi}{2 C_{\mathrm{v}}}} \\
R & =\left(n \beta-\frac{k}{C_{\mathrm{v}} \gamma_{\mathrm{w}}}\right) \\
F & =\left(\frac{\sigma_{0} m_{\mathrm{v}}}{n \beta-R}\right) \\
G & =-\left(\frac{\sigma_{0} m_{\mathrm{v}}}{n \beta-R}\right) \frac{\mathrm{e}^{-2 a h}}{\left(1+\mathrm{e}^{-2 a h}\right)} \\
H & =-\left(\frac{\sigma_{0} m_{\mathrm{v}}}{n \beta-R}\right) \frac{1}{\left(1+\mathrm{e}^{-2 a h}\right)} \\
T & =\left(\frac{\sigma_{0} m_{\mathrm{v}}}{n \beta-R}\right) n \beta h
\end{aligned}
$$

where $m_{\mathrm{v}}$ is the coefficient of compressibility; $C_{\mathrm{v}}$, the coefficient of consolidation and $\sigma_{0}$, the amplitude of cyclic loading.

Fig. 3 compares displacement, pore water pressure and effective stress obtained by the current procedure with analytical solutions at $t=120 \mathrm{~s}$ ( 6 cycles). Good agreement is observed for the displacements, pore water pressures and effective stresses. All numerical results are oscillation-free. Figs. 4 and 5 are the temporal response of displacements, pore water pressures and effective stresses. The analytical solutions are also plotted for comparison. In general, the numerical results agree well with the analytical solutions and the accuracy of numerical model is reasonably high.

\subsection{Two-dimensional transient wave-induced response in a seabed of finite thickness}

\subsubsection{Meshless model}

A two-dimensional transient problem as shown in Fig. 6 is studied under wave-induced loading. Selfweight of soil masses is not taken into consideration. This is a typical transient problem with periodic 

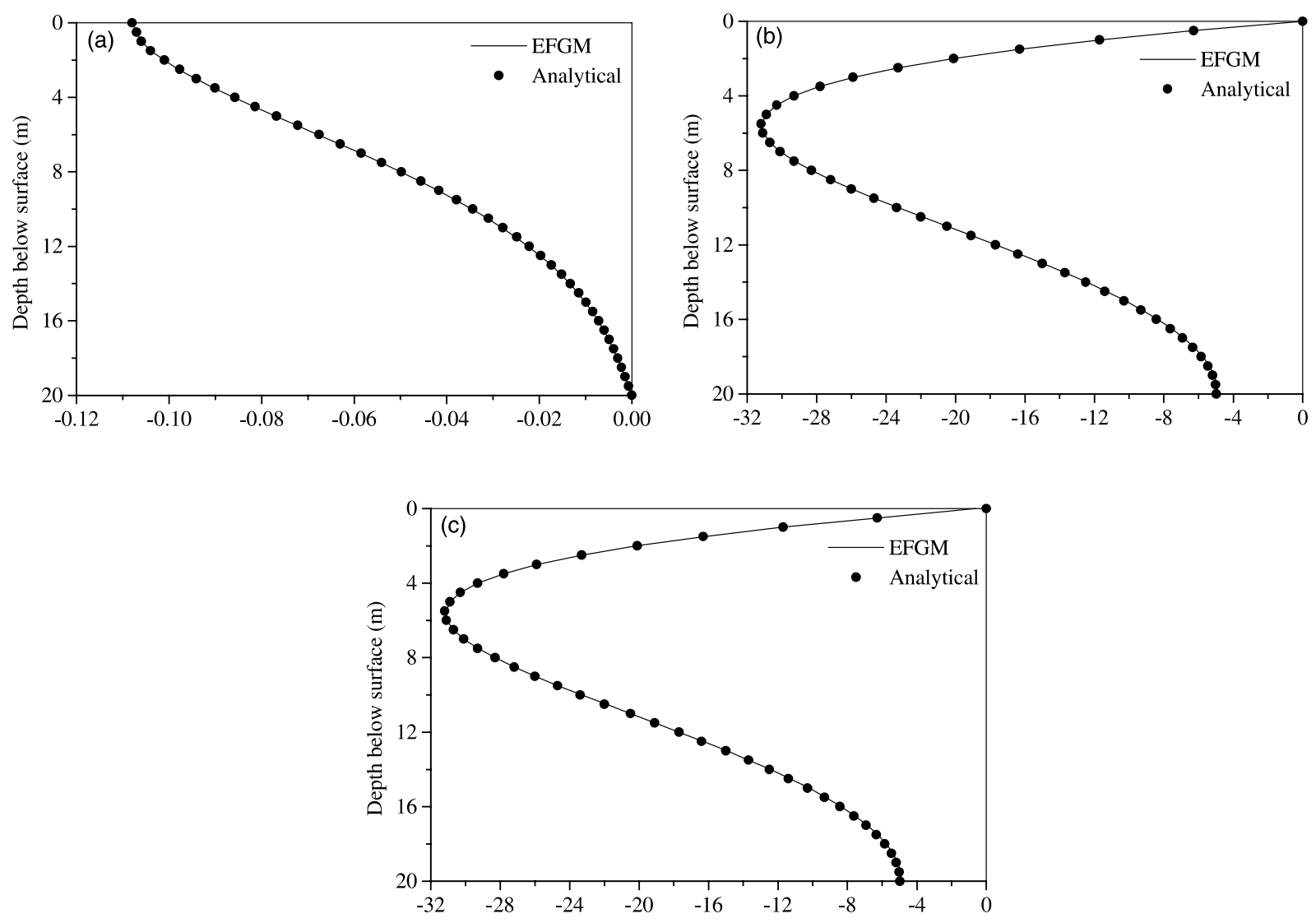

Fig. 3. Distribution of displacement, pore water pressure and effective stress at $120 \mathrm{~s}$. (a) Displacement (m), (b) pore water pressure $\left(\mathrm{kN} / \mathrm{m}^{2}\right)$ and $(\mathrm{c})$ effective stress $\left(\mathrm{kN} / \mathrm{m}^{2}\right)$.

boundary conditions in both time and space. The boundary conditions are listed as follows: The bottom $\Gamma_{\mathrm{b}}$ is fixed and impermeable. Seabed surface $\Gamma_{\mathrm{t}}$ has traction boundary $\Gamma_{\sigma}$ and pore water pressure boundary $\Gamma_{\mathrm{p}}$. If the relative acceleration between water and soils is neglected, these boundaries are expressed as follows:

$$
\begin{aligned}
& \sigma_{z}^{\prime}=0, \quad \sigma_{x z}=0 \quad \text { on } \Gamma_{\mathrm{t}} \\
& \bar{p}=p_{0} \cos (a x-\varpi t) \quad \text { on } \Gamma_{\mathrm{t}}
\end{aligned}
$$

where $a=2 \pi / l$ is the wave number; $l$, the wavelength; $\varpi=2 \pi / T$, the wave frequency; $T$, the wave period and $x$, the horizontal coordinate. The amplitude factor $p_{0}$ in Eq. (22b) is related to wave pressure. The firstorder linear wave theory (Madsen, 1976) gives

$$
p_{0}=\frac{\gamma_{\mathrm{w}} H}{2 \cosh a d_{\mathrm{w}}}
$$

in which $H$ is the wave height and $d_{\mathrm{w}}$ still water depth. The traction boundary in Eqs. (22a) and (22b) can be directly implemented through total traction on the seabed surface: 


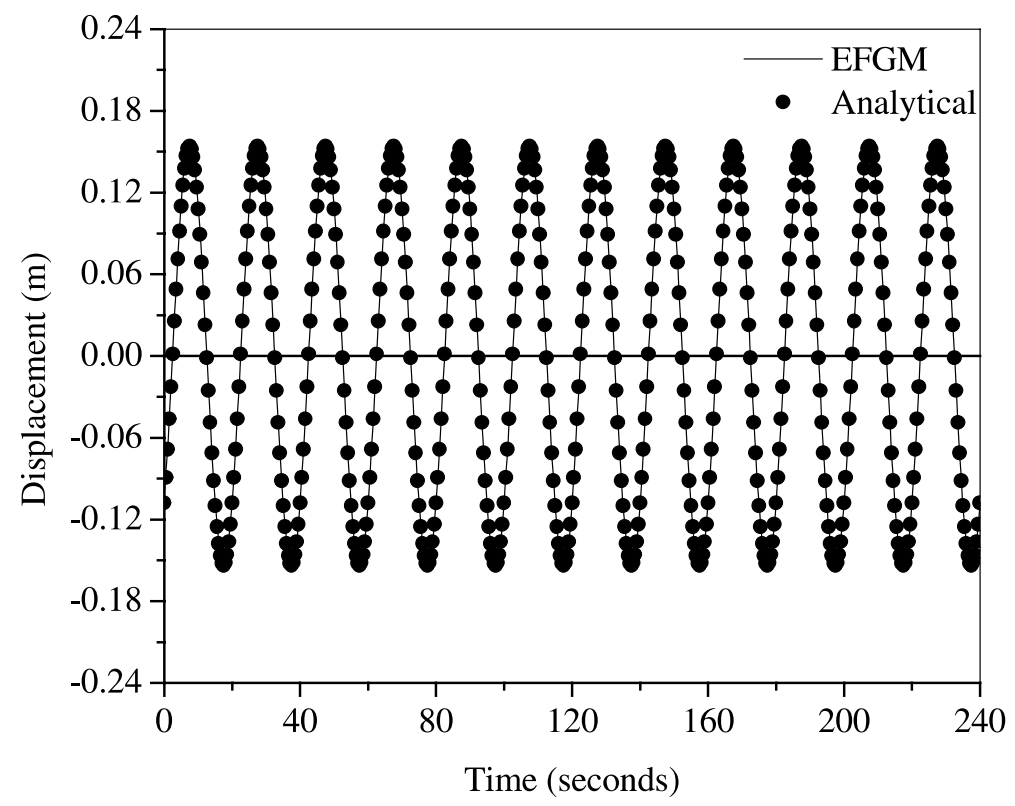

Fig. 4. Displacement history at the surface of the soil layer.
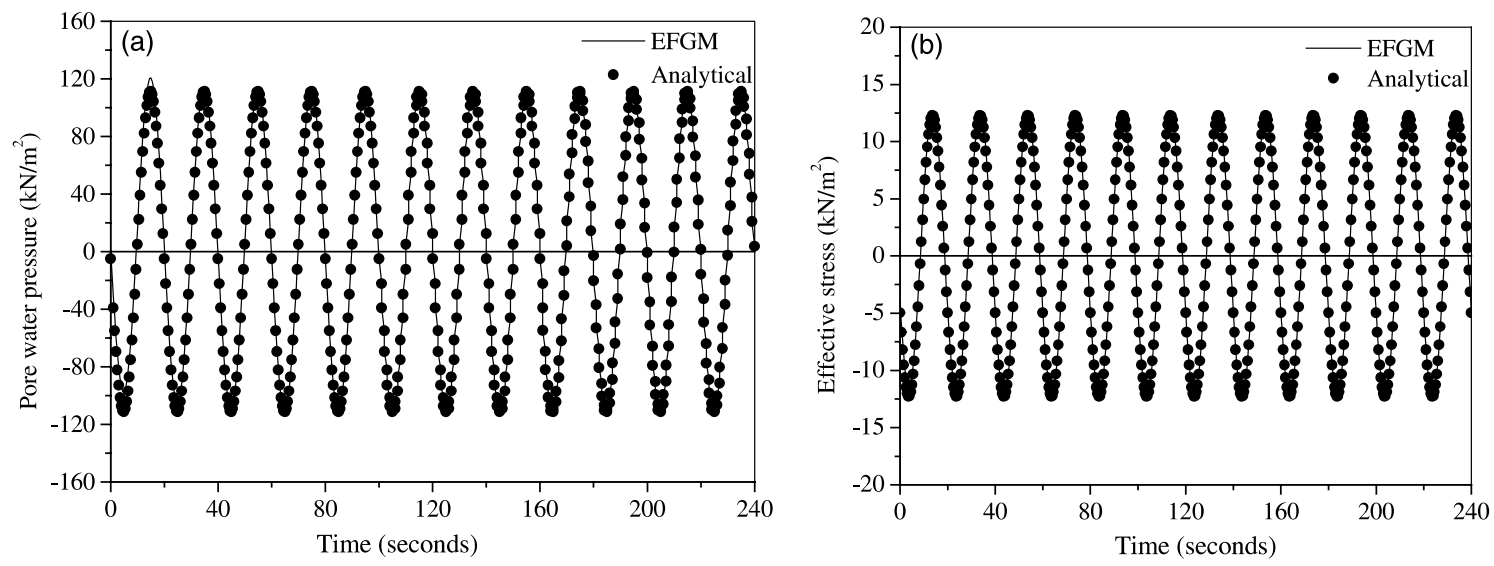

Fig. 5. History of pore water pressure and effective stress at the bottom. (a) Pore water pressure and (b) effective stress.

$$
\bar{t}=p_{0} \cos (a x-\varpi t) \quad \text { on } \Gamma_{\mathrm{t}}
$$

The same material parameters as one-dimensional case are used except the Young's modulus of soil skeleton $(E)$ chosen as $1 \times 10^{4} \mathrm{kN} / \mathrm{m}^{2}$. The $p_{0}$ in Eq. (23) is assumed to be $100 \mathrm{kN} / \mathrm{m}^{2}$. The wavelength $l$ is taken as $15 \mathrm{~m}$ and one-wavelength soil layer is taken for computation. The computation time is taken as $250 \mathrm{~s}$ ( 12.5 cycles).

Fig. 7 gives two meshless models for this problem. The regular node distribution (Fig. 7(a)) has 441 nodes and the irregular node distribution (Fig. 7(b)) has 461 nodes. The dotted lines form background cells. The irregular node distribution is used to check the effect of node distribution. Time step size is chosen as 


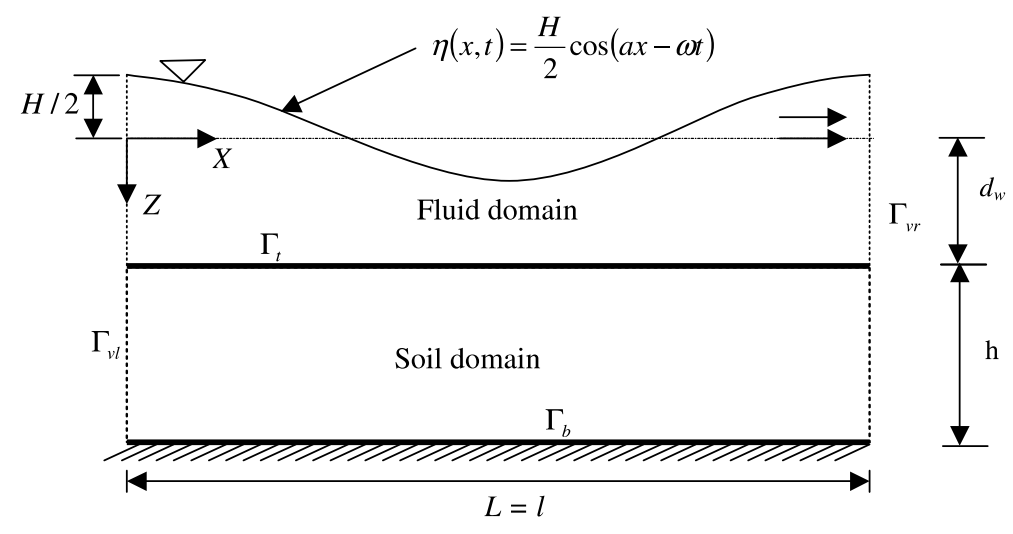

Fig. 6. A 2-D wave-induced transient problem.

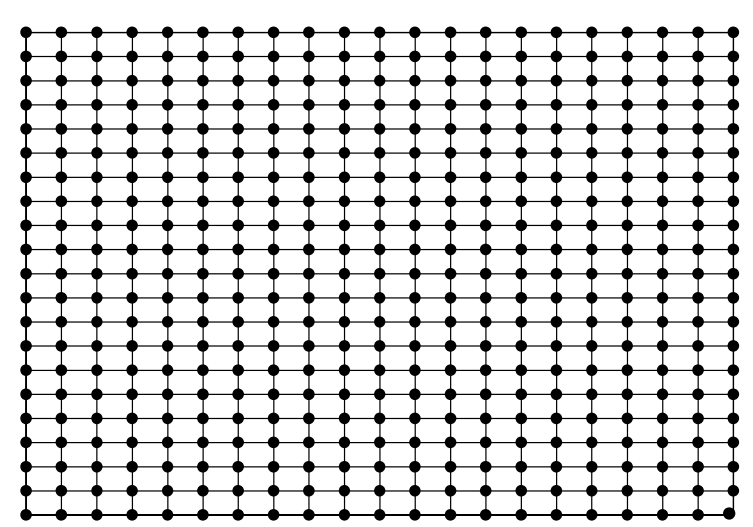

(a)

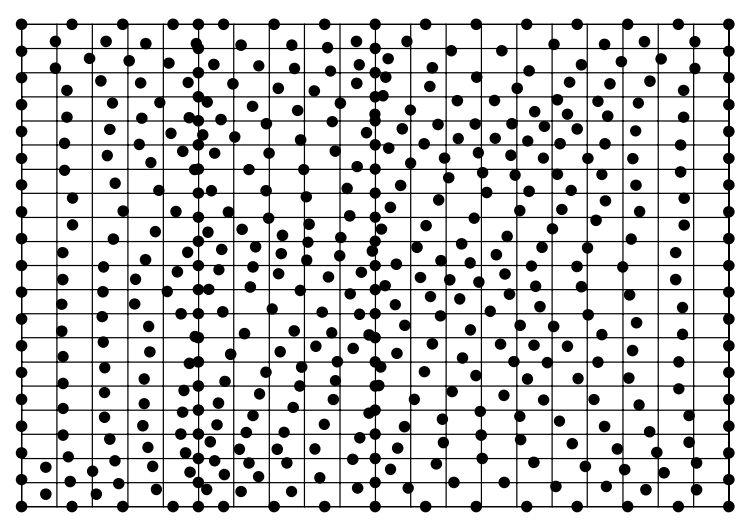

(b)

Fig. 7. Meshless model for the 2-D transient problem. (a) Regular node distribution and (b) irregular node distribution.

$0.125 \mathrm{~s}$ throughout the $250 \mathrm{~s}$ except at initial stage. At the initial stage, time step size is taken as $0.025 \mathrm{~s}$ until $0.125 \mathrm{~s}$. A total of 2004 time steps are used for the entire $250 \mathrm{~s}$.

\subsubsection{Analytical solution}

The analytical solution is the extension of Madsen (1978)'s work from infinite thickness to finite thickness. This analytical approach is different from the numerical scheme proposed by Thomas (1988). Displacements $\left(u_{z}\right.$ and $\left.u_{x}\right)$, pore water pressure $(p)$, effective stress $\left(\sigma_{z z}^{\prime}, \sigma_{x x}^{\prime}\right.$ and $\left.\sigma_{x z}^{\prime}\right)$ are obtained as

$$
\begin{aligned}
& u_{z}=\operatorname{Re}\left[\mathrm{i}\left\{\left[-\left(C_{1}-C_{2} z\right)\right] \mathrm{e}^{-a z}+\left[\left(C_{3}-C_{4} z\right)\right] \mathrm{e}^{a z}+\frac{\delta}{a}\left(C_{2} \mathrm{e}^{-a z}+C_{4} \mathrm{e}^{a z}\right)-\frac{\alpha}{a}\left(C_{5} \mathrm{e}^{-\alpha z}-C_{6} \mathrm{e}^{\alpha z}\right)\right\} \mathrm{e}^{\mathrm{i}(a x-\varpi t)}\right] \\
& u_{x}=\operatorname{Re}\left[\left\{\left(C_{1}-C_{2} z\right) \mathrm{e}^{-a z}+\left(C_{3}-C_{4} z\right) \mathrm{e}^{a z}+C_{5} \mathrm{e}^{-\alpha z}+C_{6} \mathrm{e}^{\alpha z}\right\} \mathrm{e}^{\mathrm{i}(a x-\varpi t)}\right] \\
& p=\operatorname{Re}\left[\frac{G \mathrm{i}}{1-2 v}\left\{[\delta-3+4 v]\left(C_{2} \mathrm{e}^{-a z}-C_{4} \mathrm{e}^{a z}\right)+2 a(1-v)\left[1-\frac{\alpha^{2}}{a^{2}}\right]\left(C_{5} \mathrm{e}^{-\alpha z}+C_{6} \mathrm{e}^{\alpha z}\right)\right\} \mathrm{e}^{\mathrm{i}(a x-\sigma t)}\right]
\end{aligned}
$$



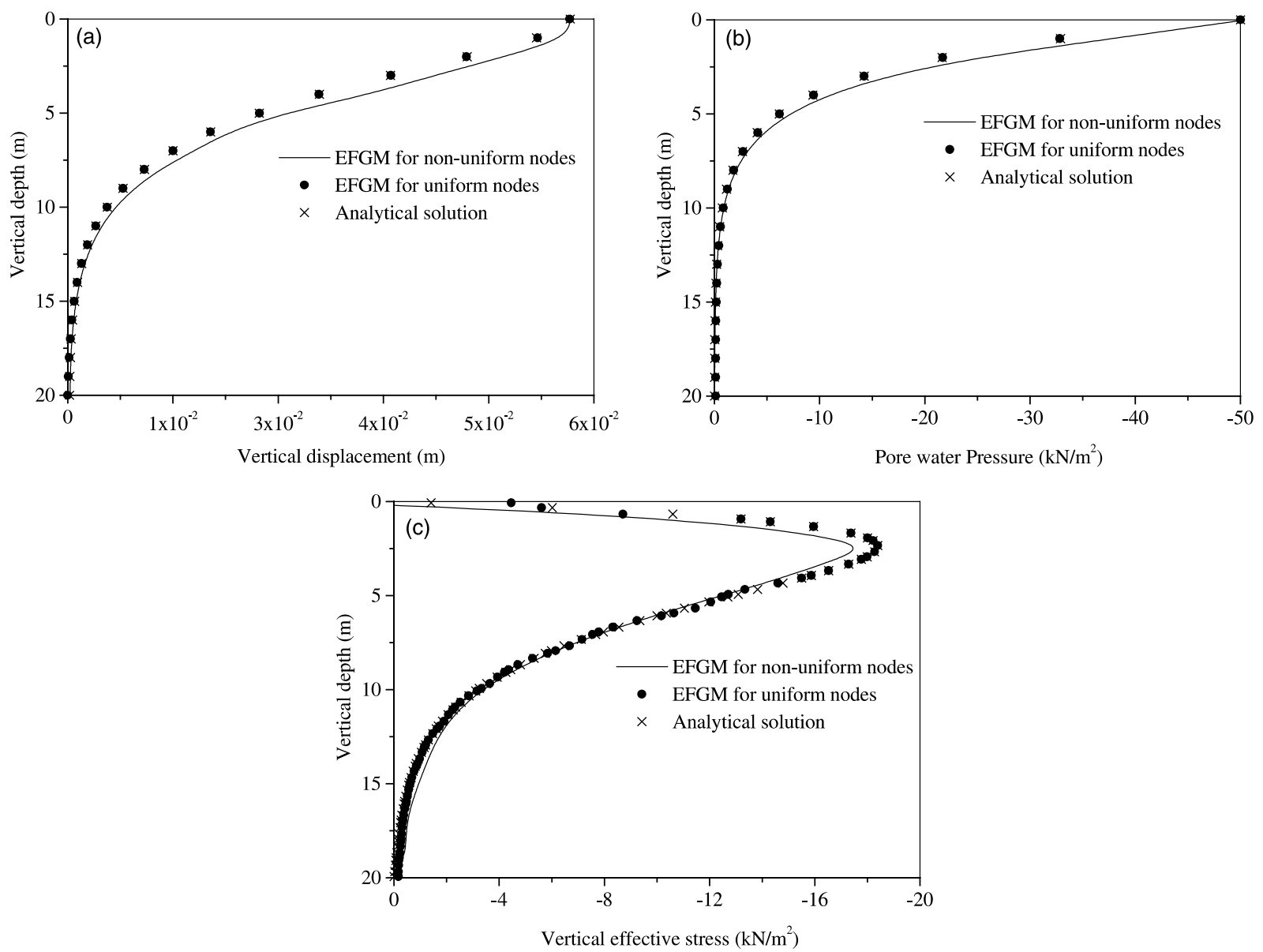

Fig. 8. Displacement, pore water pressure and effective stress along vertical section $x=7.5 \mathrm{~m}$. Distribution of (a) vertical displacement, (b) pore water pressure and (c) vertical effective stress.

$$
\begin{aligned}
& \sigma_{z z}^{\prime}=\operatorname{Re}[-\frac{2 G \mathrm{i}}{(1-2 v)}\left[\left\{-a(1-2 v)\left(C_{1}-C_{2} z\right)+(1-v)(\delta-1) C_{2}\right\} \mathrm{e}^{-a z}-\left\{a(1-2 v)\left(C_{3}-C_{4} z\right)\right.\right. \\
&+\left.\left.\left.(1-v)(\delta-1) C_{4}\right\} \mathrm{e}^{a z}+a\left(v-(1-v) \frac{\alpha^{2}}{a^{2}}\right)\left(C_{5} \mathrm{e}^{-\alpha z}+C_{6} \mathrm{e}^{\alpha z}\right)\right] \mathrm{e}^{\mathrm{i}(a x-\varpi t)}\right] \\
& \sigma_{x x}^{\prime}=\operatorname{Re}\left[-\frac{2 G \mathrm{i}}{(1-2 v)}\left[\left\{a(1-2 v)\left(C_{1}-C_{2} z\right)+v(\delta-1) C_{2}\right\} \mathrm{e}^{-a z}+\left\{a(1-2 v)\left(C_{3}-C_{4} z\right)\right.\right.\right. \\
&\left.\left.\left.-v(\delta-1) C_{4}\right\} \mathrm{e}^{a z}+a\left(1-v-v \frac{\alpha^{2}}{a^{2}}\right)\left(C_{5} \mathrm{e}^{-\alpha z}+C_{6} \mathrm{e}^{\alpha z}\right)\right] \mathrm{e}^{\mathrm{i}(a x-\varpi t)}\right] \\
& \sigma_{x z}^{\prime}=\operatorname{Re}\left[-G\left[\left\{2 a\left(C_{1}-C_{2} z\right)-(\delta-1) C_{2}\right\} \mathrm{e}^{-a z}-\left\{2 a\left(C_{3}-C_{4} z\right)+(\delta-1) C_{4}\right\} \mathrm{e}^{a z}\right.\right. \\
&\left.\left.+2 \alpha C_{5} \mathrm{e}^{-\alpha z}-2 \alpha C_{6} \mathrm{e}^{\alpha z}\right] \mathrm{e}^{\mathrm{i}(a x-\varpi t)}\right]
\end{aligned}
$$



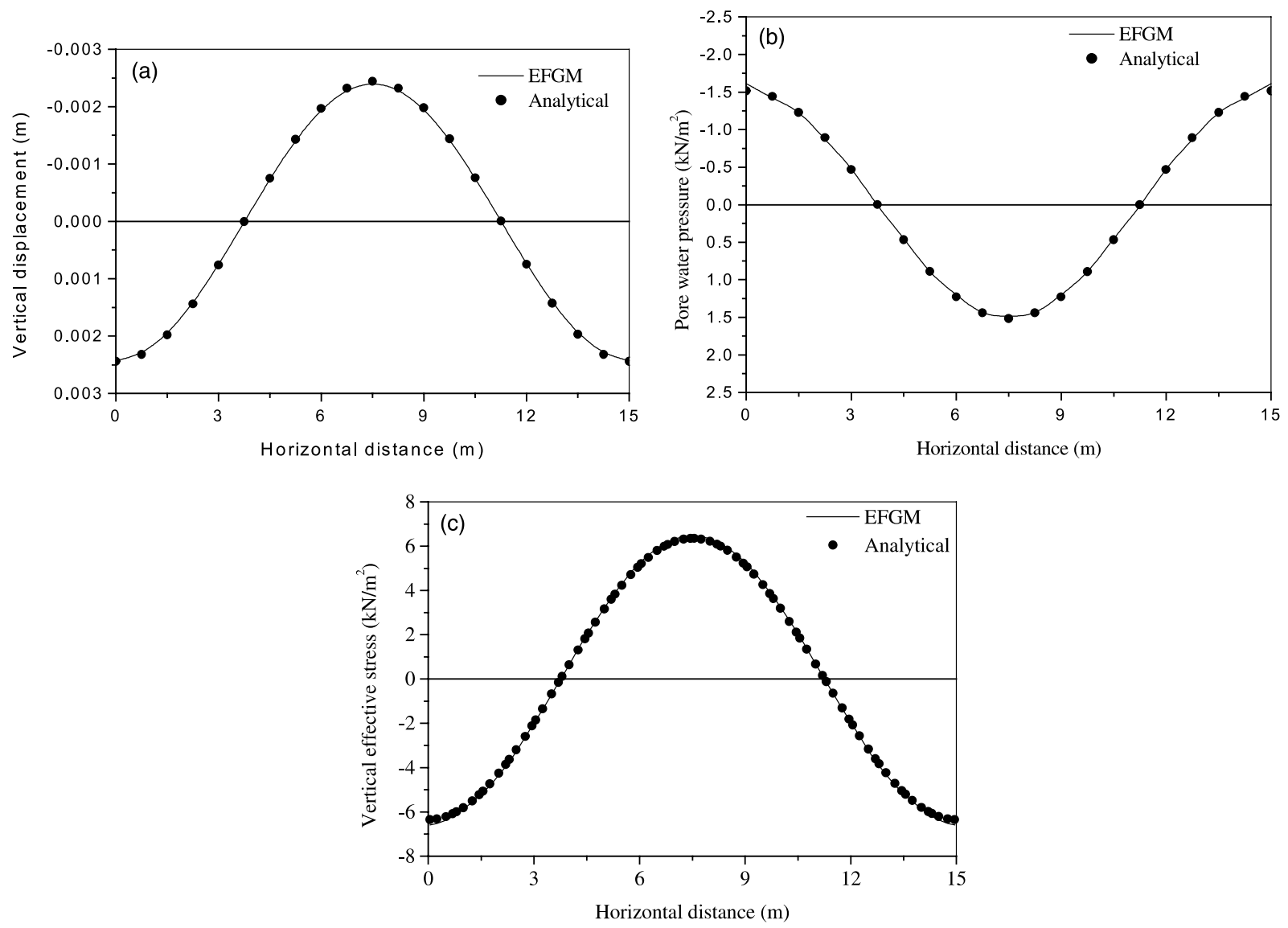

Fig. 9. Displacement, pore water pressure and effective stress along horizontal section at $z=10 \mathrm{~m}$. (a) Vertical displacement, (b) pore water pressure and (c) vertical effective stress.

Above constants $C_{1}, C_{2}, C_{3}, C_{4}, C_{5}$ and $C_{6}$ satisfy following six linear equations:

$$
\begin{aligned}
& a(1-2 v)\left(C_{1}+C_{3}\right)-(1-v)(\delta-1)\left(C_{2}-C_{4}\right)-a\left(v-(1-v) \frac{\alpha^{2}}{a^{2}}\right)\left(C_{5}+C_{6}\right)=0 \\
& 2 a C_{1}-(\delta-1)\left(C_{2}+C_{4}\right)-2 a C_{3}+2 \alpha C_{5}-2 \alpha C_{6}=0 \\
& {[\delta-3+4 v]\left(C_{2}-C_{4}\right)+2 a(1-v)\left[1-\frac{\alpha^{2}}{a^{2}}\right]\left(C_{5}+C_{6}\right)=\frac{p_{0}(1-2 v)}{G \mathrm{i}}} \\
& C_{1} \mathrm{e}^{a h}+h C_{2} \mathrm{e}^{a h}+C_{3} \mathrm{e}^{-a h}+h C_{4} \mathrm{e}^{-a h}+C_{5} \mathrm{e}^{\alpha h}+C_{6} \mathrm{e}^{-\alpha h}=0 \\
& -C_{1} \mathrm{e}^{a h}+\left(\frac{\delta}{a}-h\right) C_{2} \mathrm{e}^{a h}+C_{3} \mathrm{e}^{-a h}-\left(\frac{\delta}{a}+h\right) C_{4} \mathrm{e}^{-a h}-\frac{\alpha}{a}\left(C_{5} \mathrm{e}^{\alpha h}-C_{6} \mathrm{e}^{-\alpha h}\right)=0 \\
& a[\delta-3+4 v]\left(C_{2} \mathrm{e}^{a h}+C_{4} \mathrm{e}^{-a h}\right)+2 \alpha(1-v) a\left[1-\frac{\alpha^{2}}{a^{2}}\right]\left(C_{5} \mathrm{e}^{\alpha h}-C_{6} \mathrm{e}^{-\alpha h}\right)=0
\end{aligned}
$$



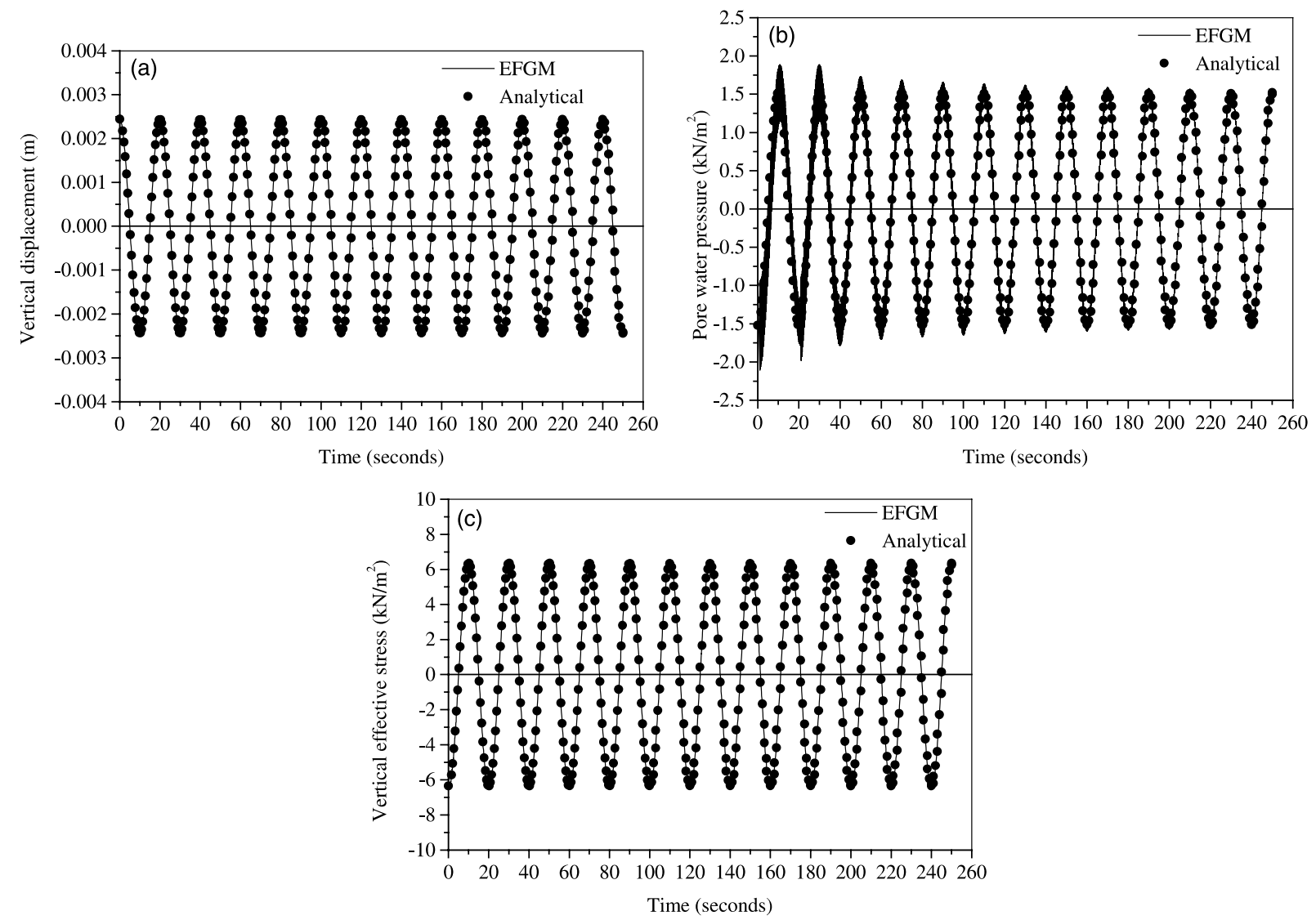

Fig. 10. History of displacement, pore water pressure and effective stress at the point $(x, z)=(7.5,10)$. (a) Vertical displacement, (b) pore water pressure and (c) vertical effective stress.

where the parameters are defined as follows:

$$
\begin{aligned}
& \delta=\frac{(3-4 v) n \beta+\frac{1-2 v}{G}}{n \beta+\frac{1-2 v}{G}} \\
& \alpha=\sqrt{a^{2}-\kappa^{2}} \\
& \kappa^{2}=\frac{\mathrm{i} \varpi \gamma_{\mathrm{w}}}{k}\left(n \beta+m_{\mathrm{v}}\right) \\
& m_{\mathrm{v}}=\frac{(1-2 v)(1+v)}{E(1-v)}
\end{aligned}
$$

\subsubsection{Comparison of numerical results with analytical solution}

Fig. 8 compares the numerical results with analytical solutions for displacements, pore water pressures, effective stresses respectively at $t=150 \mathrm{~s}$ ( 7.5 cycles). From these figures, it is found that nodal distributions have little effect on the numerical results of the current procedure. However, the accuracy is reasonably 
acceptable even for irregular node distribution. The deviation between regular and irregular node distributions can be further reduced if sufficient nodes are enclosed in an influence domain. Numerical results have no oscillation regardless of node distributions. This characteristic is not readily achievable in FEM.

Fig. 9 shows the distribution of displacement, pore water pressure and effective stress along horizontal section $z=10 \mathrm{~m}$ (mid-depth) (regular node distribution) and at $t=250 \mathrm{~s}$ (12.5 cycles). The agreement between the EFGM and analytical solutions are satisfactory. All numerical results are oscillation-free. Fig. 10 compares the temporal response of displacement, pore water pressure, effective stress at the point $(x, z)=(7.5,10)$. The EFGM and analytical solutions agree very well again.

As a summary, the current procedure can simulate the transient responses of the seabed whether node distributions are regular or not. The regular node distribution has higher accuracy while the irregular node distribution still has reasonable accuracy. The EFGM may be an oscillation-free algorithm even the CrankNicolson algorithm is used in time domain. However, the FEM has difficulty in choosing a suitable time step size with oscillation-free. This is an advantage of the current method over FEM.

\section{Moving boundary problem}

This problem checks the capability of the EFGM for moving boundary problems. An impermeable mass-less body of infinite length is moving along the top surface of the seabed at a constant speed. The seabed is subjected to wave-induced load. The top surface is permeable except the boundary enclosed by the impermeable body. The both side boundaries are still assumed to be periodic. Fig. 11 gives the meshless models at the various time steps. The nodes have uniformly distributed fixed nodes ( 461 hollow-circle nodes) as well as non-uniform moving nodes (the filled-circle nodes). The moving nodes are changing in numbers and positions with time, depending on the position of the impermeable body (as noted by the dark straight line on the top of the soil surface). The impermeable body moves at a constant speed of $0.05 \mathrm{~m} / \mathrm{s}$. Dashed lines form background cells which remain unchanged during the whole computation.

Computational parameters for seabed soil and wave are the same as those in Section 4.2. At any time step, non-uniform moving nodes are positioned over an assumed highly variable zone of dimensions of $\left(l_{1}+l_{2}\right)$ and $h_{1}$ along horizontal and vertical directions respectively. $l_{1}$ is taken as $2 \mathrm{~m}$ or distance of the edge of the impermeable body from left boundary whichever is smaller, $l_{2}$ is taken as $2 \mathrm{~m}$ or distance of the edge of the impermeable body from right boundary whichever is smaller, and $h_{1}$ is taken as $5 \mathrm{~m}$ down from $0.25 \mathrm{~m}$ level from the top soil surface. Depending on the zone size, the number and position of the moving nodes are determined by an automatic node generator. The number of moving nodes varies from 32 to 55 in this example.

Soil responses are recorded only at the fixed nodes throughout the whole computation. The moving nodes are locally introduced at any time step to give higher density of nodes within the influence domain of the fixed nodes. This improves the accuracy of interpolation within that local zone. When a node is added at any time step, the responses at that node in the immediate previous time step is interpolated through the approximations given by Eqs. (17a) and (17b). The nodal indexes are then calculated as

$$
u_{j m}=\left(\sum_{I=1}^{n_{\mathrm{f}}} \phi_{\mathrm{f} I} u_{\mathrm{f} I}-\sum_{I=2}^{n_{\mathrm{f}}} \phi_{\mathrm{t} I} u_{\mathrm{f} I}\right) \phi_{j}
$$

where $u_{m}$ is the nodal index at the newly added node; $u_{\mathrm{f}}$, the nodal index at the fixed node; $\phi_{\mathrm{f}}$, the shape function determined against only for fixed nodes; $\phi_{\mathrm{t}}$, the shape function determined against for both fixed and new (or moving) nodes.

Fig. 12 gives the contour of pore water pressures at different times. The distribution of the cyclic loading applied on the top at the respective time is also shown. The dark straight line shows the position of the 
(a)

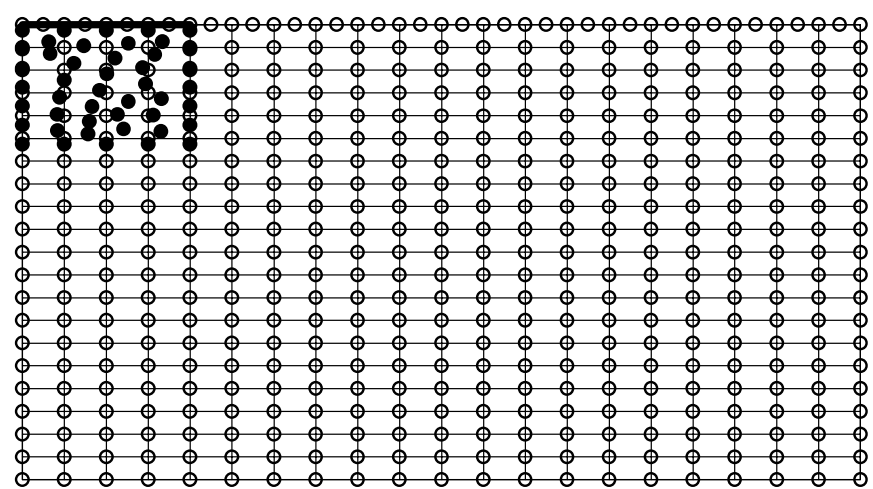

(b)

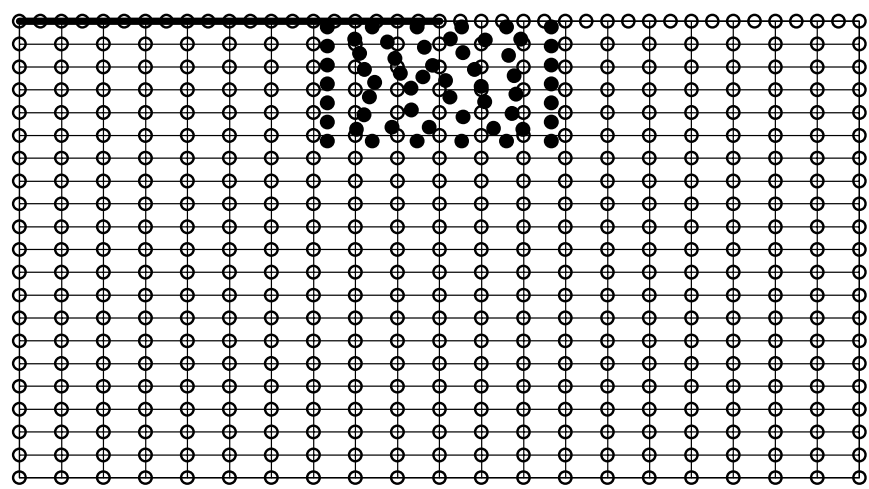

(c)

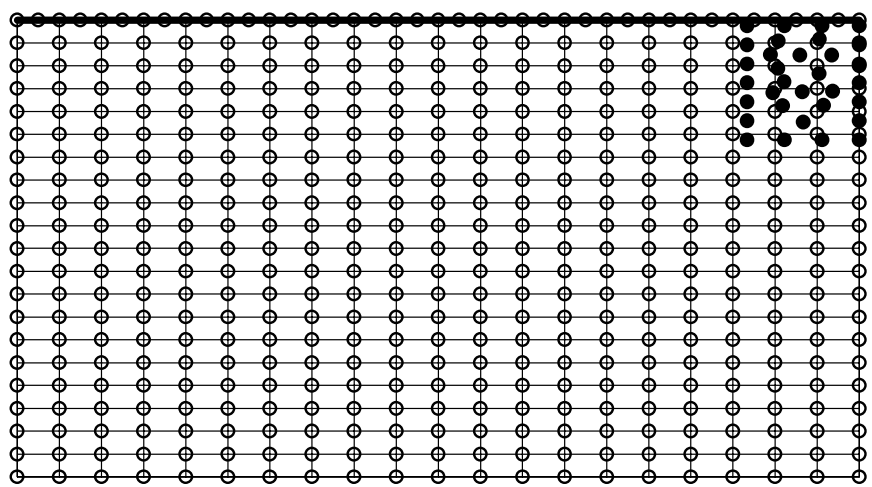

Fig. 11. Meshless models at various time steps for moving boundary conditions. (a) At 482 nd step (when top surface becomes impermeable up to $3 \mathrm{~m}$ from left). (b) At 1202nd step (when top surface becomes impermeable up to $7.5 \mathrm{~m}$ from left). (c) At 2402nd step (when whole top surface becomes impermeable).

impermeable body. These contours show that the movement of the impermeable body affects the soil responses considerably. The EFGM can easily simulate such kind of problems. This is because the movement and position of the moving nodes are easily accumulated within the fixed nodes and, as the nodes are independent of the background cells for integrations, no extra process is involved in carrying out the whole 

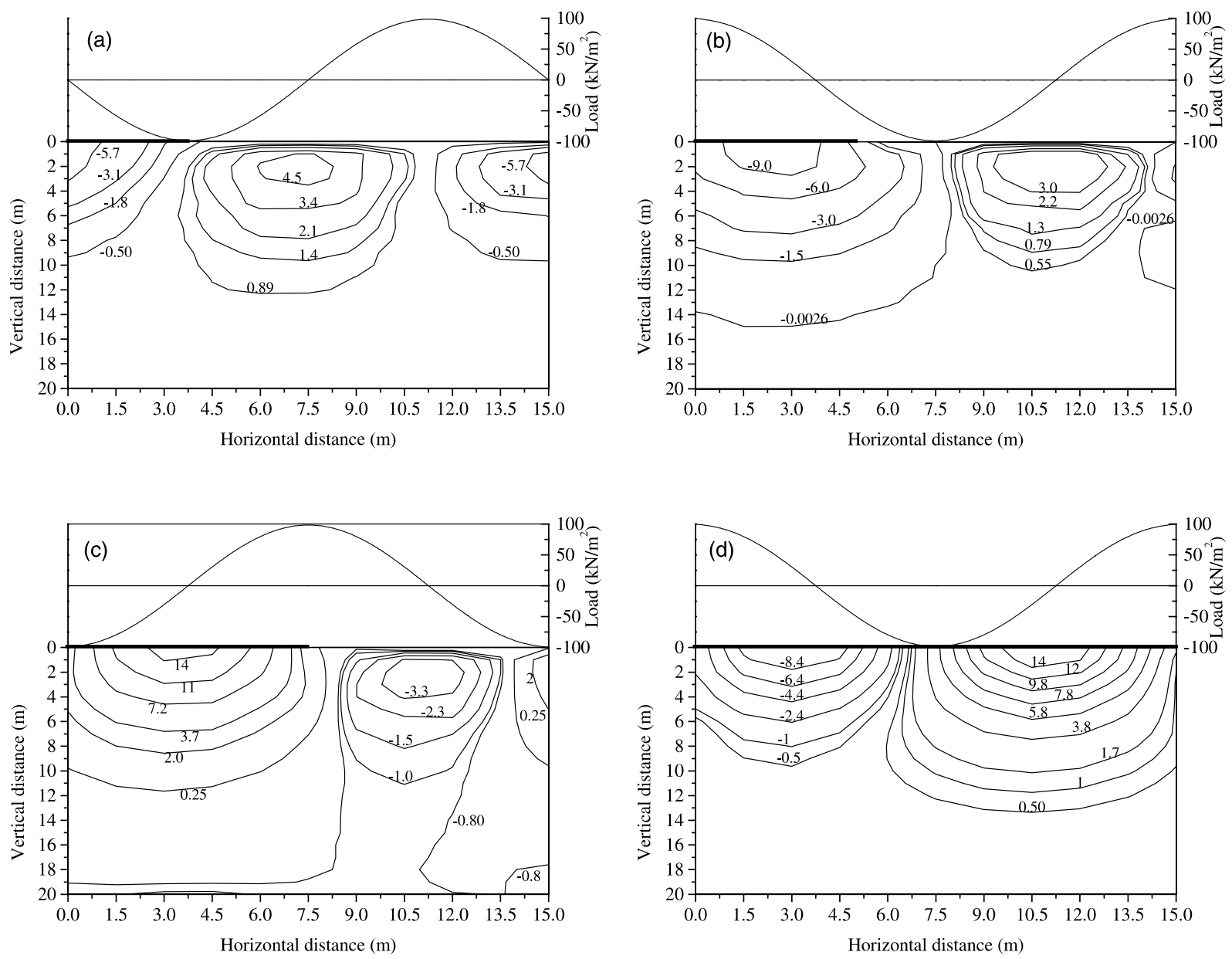

Fig. 12. Contours of pore water pressures with moving body after (a) $75 \mathrm{~s}$, (b) $100 \mathrm{~s}$, (c) $150 \mathrm{~s}$ and (d) $300 \mathrm{~s}$.

numerical procedures. FEM requires meshes and their connectivity, and the update of mesh is complex and time-consuming. Therefore, this is not an easy task for FEM.

\section{Conclusions}

A two-dimensional numerical procedure is presented based on the EFGM to analyse the transient response of saturated porous elastic soil under wave-induced loading. In this procedure, displacement and excess pore water pressure are approximated using the same shape functions constructed by MLS approximants. Lagrange multipliers are employed to implement essential and periodic boundary conditions. A one-dimensional example is designed for temporal periodic conditions and a two-dimensional example for both temporal and spatial periodic conditions. Analytical solutions are developed for the finite thickness of soils to check the accuracy of the current numerical procedure. Finally, the moving boundary problem is designed to check its capability. 
The current procedure revised variational formulations to incorporate temporal and spatial periodic boundary conditions. Whether the problems are one-dimensional or two-dimensional, the current procedure provides very accurate prediction of the transient soil response.

The current procedure performs very well not only for regular node distributions but also for irregular node distributions. Irregular node distribution has lower accuracy but the accuracy is acceptable. The numerical results are oscillation-free, while FEM has difficulty to get oscillation-free results for the same time step size. The current procedure is easy and effective to treat moving boundary problems. This is because the EFGM has the flexibility to add or remove nodes easily from the discretized domain. This is an advantage over the FEM.

Analytical solutions are developed in this paper to check the accuracy of the current procedure. This solution is the extension of Madsen (1978)'s work from infinite soil thickness to finite thickness. The comparison between analytical solutions and numerical results show that the current procedure has high accuracy for both temporal and spatial periodic problems like wave-induced transient responses.

As a numerical method, the current procedure can be easily extended to the anisotropic, non-homogeneous seabed. This paper just regards water wave as a periodic loading on the seabed although the seabed and water wave interaction is an interesting topic.

\section{Acknowledgements}

This study was financially supported by the US Office of Navy Research under grant number N0001401-1-0457. The authors also thank the valuable comments from reviewers.

\section{Appendix A}

After the interpolation in Eqs. (17a)-(17f) are introduced into the weak forms of Eqs. (16a)-(16f) and the time integration Eq. (18) is applied, the discrete system equations are obtained as follows:

$$
\begin{aligned}
& K_{I J} u_{J}^{t+1}+L_{I J} p_{J}^{t+1}+G_{I K} \lambda_{1 K}^{t+1}+\left(G_{I K}^{\mathrm{vl}}-G_{I K}^{\mathrm{vr}}\right) \lambda_{3 K}^{t+1}=f_{u I} \\
& L_{I J}^{\mathrm{T}} u_{J}^{t+1}+\left(H_{I J} \Delta t \theta-M_{I J}\right) p_{J}^{t+1}+G_{I K}^{\prime} \Delta t \theta \lambda_{2 K}^{t+1}+\Delta t \theta\left(G_{I K}^{\mathrm{vl}}-G_{I K}^{\mathrm{vr}}\right) \lambda_{4 K}^{t+1} \\
& \quad=L_{I J}^{\mathrm{T}} u_{J}^{t}-M_{I J} p_{J}^{t}+\Delta t f_{p I}-H_{I J} \Delta t(1-\theta) p_{J}^{t}-G_{I K}^{\prime} \Delta t(1-\theta) \lambda_{2 K}^{t}-\Delta t(1-\theta)\left(G_{I K}^{\mathrm{vl}}-G_{I K}^{\mathrm{vr}}\right) \lambda_{4 K}^{t+1} \\
& G_{I K}^{\mathrm{T}} u_{K}^{t+1}=f_{\lambda_{1} I} \\
& G_{I K}^{\mathrm{T}} p_{K}^{t+1}=f_{\lambda_{2} I} \\
& \left(G_{I K}^{\mathrm{vIT}}-G_{I K}^{\mathrm{vrT}}\right) u_{K}^{t+1}=0 \\
& \left(G_{I K}^{\mathrm{vI} \mathrm{T}^{\mathrm{T}}}-G_{I K}^{\prime \mathrm{vrT}}\right) p_{K}^{t+1}=0
\end{aligned}
$$

The matrix form for above equations is as follows:

$$
[R]\left[S^{t+1}\right]=[F]+[Q]\left[S^{t}\right]
$$

where $[R],\left[S^{t+1}\right],[F],[Q]$ and $\left[S^{t}\right]$ are given by 


$$
\begin{aligned}
& {[R]=\left[\begin{array}{cccccc}
K & L & G & 0 & \left(G^{\mathrm{vl}}-G^{\mathrm{vr}}\right) & 0 \\
L^{\mathrm{T}} & (\theta \Delta t H-M) & 0 & \theta \Delta t G^{\prime} & 0 & \left(G^{\prime \mathrm{vl}}-G^{\prime \mathrm{vr}}\right) \Delta t \theta \\
G^{\mathrm{T}} & 0 & 0 & 0 & 0 & 0 \\
0 & G^{\mathrm{T}} & 0 & 0 & 0 & 0 \\
\left(G^{\mathrm{vlT}}-G^{\mathrm{vrT}}\right) & 0 & 0 & 0 & 0 & 0 \\
0 & \left(G^{\prime \mathrm{vlT}}-G^{\prime \mathrm{vrT}}\right) & 0 & 0 & 0 & 0
\end{array}\right]} \\
& {[Q]=\left[\begin{array}{cccccc}
0 & 0 & 0 & 0 & 0 & 0 \\
L^{\mathrm{T}} & -(\Delta t(1-\theta) H+M) & 0 & -\Delta t(1-\theta) G^{\prime} & 0 & -\Delta t\left(G^{\prime \mathrm{vl}}-G^{\mathrm{vr}}\right)(1-\theta) \\
0 & 0 & 0 & 0 & 0 & 0 \\
0 & 0 & 0 & 0 & 0 & 0 \\
0 & 0 & 0 & 0 & 0 & 0 \\
0 & 0 & 0 & 0 & 0 & 0
\end{array}\right]} \\
& {\left[S^{t+1}\right]^{\mathrm{T}}=\left[\begin{array}{llllll}
u^{t+1} & p^{t+1} & \lambda_{1}^{t+1} & \lambda_{2}^{t+1} & \lambda_{3}^{t+1} & \lambda_{4}^{t+1}
\end{array}\right]} \\
& {\left[S^{t}\right]^{\mathrm{T}}=\left[\begin{array}{llllll}
u^{t} & p^{t} & \lambda_{1}^{t} & \lambda_{2}^{t} & \lambda_{3}^{t} & \lambda_{4}^{t}
\end{array}\right]} \\
& {[F]^{\mathrm{T}}=\left[\begin{array}{llllll}
f_{u} & \Delta t f_{\mathrm{p}} & f_{\lambda_{1}} & f_{\lambda_{2}} & 0 & 0
\end{array}\right]}
\end{aligned}
$$

The superscript $(t+1)$ denotes the current time $(t+\Delta t)$. Other notations include

$$
\begin{aligned}
K_{I J} & =\int_{\Omega} B_{I}^{\mathrm{T}} D B_{J} \mathrm{~d} \Omega \\
L_{I J} & =\int_{\Omega} \phi_{I} A_{J} \mathrm{~d} \Omega \\
M_{I J} & =n \beta \int_{\Omega} \phi_{I} \phi_{J} \mathrm{~d} \Omega \\
H_{I J} & =\frac{k}{\gamma_{\mathrm{w}}} \int_{\Omega} A_{I}^{\mathrm{T}} A_{J} \mathrm{~d} \Omega \\
G_{I K} & =\int_{\Gamma_{u}} \bar{N}_{K} \phi_{I} \mathrm{~d} \Gamma \\
G_{I K}^{\prime} & =\int_{\Gamma_{\mathrm{p}}} \bar{N}_{K}^{\prime} \phi_{I} \mathrm{~d} \Gamma \\
G_{I K}^{\mathrm{vl}} & =\int_{\Gamma_{\mathrm{vl}}} \bar{N}_{K} \phi_{I} \mathrm{~d} \Gamma \\
G_{I K}^{\mathrm{vr}} & =\int_{\Gamma_{\mathrm{vr}}} \bar{N}_{K} \phi_{I} \mathrm{~d} \Gamma \\
G_{I K}^{\mathrm{vl}} & =\int_{\Gamma_{\mathrm{vl}}} \bar{N}_{K}^{\prime} \phi_{I} \mathrm{~d} \Gamma
\end{aligned}
$$




$$
\begin{aligned}
& G_{I K}^{\mathrm{vr}}=\int_{\Gamma_{\mathrm{vr}}} \bar{N}_{K}^{\prime} \phi_{I} \mathrm{~d} \Gamma \\
& f_{u I}=\int_{\Gamma_{\sigma}} \bar{t} \phi_{I} \mathrm{~d} \Gamma+\int_{\Omega} b \phi_{I} \mathrm{~d} \Omega \\
& f_{\mathrm{p} I}=\int_{\Gamma_{\varphi}} \bar{\varphi} \phi_{I} \mathrm{~d} \Gamma \\
& f_{\lambda_{1} I}=\int_{\Gamma_{u}} \bar{N}_{K} \bar{u} \mathrm{~d} \Gamma \\
& f_{\lambda_{2} I}=\int_{\Gamma_{\mathrm{p}}} \bar{N}_{K}^{\prime} \bar{p} \mathrm{~d} \Gamma \\
& B_{I}=\partial\left(\begin{array}{cc}
\left.\phi_{I}\right)= \\
\phi_{I, x} & 0 \\
0 & \phi_{I, z} \\
\phi_{I, z} & \phi_{I, x}
\end{array}\right] \\
& A_{J}=\left[\begin{array}{c}
\phi_{I, x} \\
\phi_{I, z}
\end{array}\right] \\
& \bar{N}_{K}=\left[\begin{array}{cc}
N_{k} & 0 \\
0 & N_{k}
\end{array}\right] \\
& \bar{N}_{K}^{\prime}=\left[\begin{array}{ll}
N_{k}
\end{array}\right] \\
&
\end{aligned}
$$

For one-dimensional problem, above matrices are simplified into:

$$
\begin{aligned}
& {[R]_{\text {onedi }}=\left[\begin{array}{cccc}
K & L & G & 0 \\
L^{\mathrm{T}} & (\theta \Delta t H-M) & 0 & \theta \Delta t G^{\prime} \\
G^{\mathrm{T}} & 0 & 0 & 0 \\
0 & G^{\mathrm{T}} & 0 & 0
\end{array}\right]} \\
& {[Q]_{\text {onedi }}=\left[\begin{array}{cccc}
0 & 0 & 0 & 0 \\
L^{\mathrm{T}} & -(\Delta t(1-\theta) H+M) & 0 & -\Delta t(1-\theta) G^{\prime} \\
0 & 0 & 0 & 0 \\
0 & 0 & 0 & 0
\end{array}\right]} \\
& {\left[S^{t+1}\right]_{\text {onedi }}^{\mathrm{T}}=\left[\begin{array}{llll}
u^{t+1} & p^{t+1} & \lambda_{1}^{t+1} & \lambda_{2}^{t+1}
\end{array}\right]} \\
& {\left[S^{t}\right]_{\text {onedi }}^{\mathrm{T}}=\left[\begin{array}{llll}
u^{t} & p^{t} & \lambda_{1}^{t} & \lambda_{2}^{t}
\end{array}\right]} \\
& {[F]_{\text {onedi }}^{\mathrm{T}}=\left[\begin{array}{llll}
f_{u} & \Delta t f_{\mathrm{p}} & f_{\lambda_{1}} & f_{\lambda_{2}}
\end{array}\right]}
\end{aligned}
$$

\section{References}

Babuska, I., Melenk, J.M., 1997. Partition of unity method. Int. J. Numer. Meth. Eng. 40 (4), 727-758.

Belytschko, T., Lu, Y.Y., Gu, L., 1994. Element-free Galerkin methods. Int. J. Numer. Meth. Eng. 37, $229-256$. 
Belytschko, T., Krongauz, Y., Organ, D., Fleming, M., Krysl, P., 1996. Meshless methods: an overview and recent developments. Comput. Meth. Appl. Mech. Eng. 139, 3-47.

Biot, M.A., 1941. General theory of three-dimensional consolidation. J. Appl. Phys. 12, 155-164.

Dolbow, J., Belytschko, T., 1998. An introduction to programming the meshless element free Galerkin method. Arch. Comput. Meth. Eng. 5 (3), 207-241.

Duarte, C.A., Oden, J.T., 1996. $H-p$ clouds - an $h-p$ meshless method. Numer. Meth. Partial Differential Equations 12, $673-705$.

Ghaboussi, J., Dikman, S.U., 1978. Liquefaction analysis of horizontally layered sands. J. Geotech. Eng. Division ASCE GT3, 341-356.

Ghaboussi, J., Wilson, E.L., 1972. Variational formulation of dynamics of fluid-saturated porous elastic solids. J. Eng. Mech. Division ASCE EM4, 947-963.

Ghaboussi, J., Wilson, E.L., 1973. Seismic analysis of earth dam-reservoir systems. J. Soil Mech. Found. Division ASCE SM10, $849-862$.

Karim, M.R., Wang, J.G., Nogami, T., 2001. Element-free Galerkin method: a meshless discretization approach in consolidation analysis. In: Proceedings of International Conference on Computation Mechanics-New Frontiers for New Millennium. Elsevier Science, pp. 953-958.

Lancaster, P., Salkauskas, K., 1981. Surfaces generated by moving least squares methods. Math. Comput. 37, $141-158$.

Liu, W.K., Jun, S., Zhang, Y.F., 1995. Reproducing kernel particle methods. Int. J. Numer. Meth. Fluids 20, $1081-1106$.

Madsen, O.S., 1976. Wave climate of the continental margin: elements of its mathematical description. In: Stanley, D.F., Swift, D.J.P. (Eds.), Marine Sediment Transport and Environmental Management. John Wiley, New York, pp. 65-90.

Madsen, O.S., 1978. Wave-induced pore pressure and effective stresses in a porous bed. Geotechnique 28 (4), $377-393$.

Modaressi, H., Aubert, P., 1996. A diffuse element-finite element technique for transient coupled analysis. Int. J. Numer. Meth. Eng. 39 (22), 3809-3838.

Monaghan, J.J., 1988. An introduction to SPH. Comput. Phys. Commun. 48, 89-96.

Okusa, S., 1985. Wave-induced stresses in unsaturated submarine sediments. Geotechnique 35 (4), 517-532.

Prevost, J.H., 1982. Nonlinear transient phenomena in saturated porous media. Comput. Meth. Appl. Mech. Eng. $20,3-18$.

Sandhu, R.S., Wilson, E.L., 1969. Finite element analysis of seepage in elastic media. Proceedings of ASCE, EM, 95.

Thomas, S.D., 1988. A finite element model for the analysis of wave-induced stresses and pore pressures in a variably saturated seabed. Soil Mechanics Report no. SM087/88, University of Oxford.

Verruijt, A., 1969. Elastic storage of aquifers. In: De Wiest, R.J.M. (Ed.), Flow through Porous Media. Academic Press, New York, pp. 331-376.

Wang, J.G., Liu, G.R., 2002. A point interpolation meshless method based on radial basis functions. Int. J. Numer. Meth. Eng. 54, $1623-1648$.

Wang, J.G., Liu, G.R., Lin, P., 2002. Numerical analysis of Biot's consolidation process by radial point interpolation method. Int. J. Solids Struct. 39 (6), 1557-1573.

Yamamoto, T., Koning, H.L., Sellmeijer, H., Hijum, E.V., 1978. On the response of a poro-elastic bed to water waves. J. Fluid Mech. 87 (part 1), 193-206.

Zeinkiewicz, O.C., 1980. Nonlinear problems of soil statics and dynamics. In: Proceedings of Europe-US Symposium on Nonlinear Finite Element Analysis in Structural Mechanics. Ruhr Universitat, Bochum, Springer-Verlag.

Zeinkiewicz, O.C., Shiomi, T., 1984. Dynamic behavior of saturated porous media; the generalised Biot formulation and its numerical solution. Int. J. Numer. Anal. Meth. Geomech. 8, 71-96.

Zeinkiewicz, O.C., Humpheson, C., Lewis, R.W., 1977. A unified approach to soil mechanics problems. In: Finite Elements in Geomechanics. John Willy, New York, pp. 151-178. 